\title{
Visualizing Astigmatism in the SEM Electron Probe
}

\author{
Mandy C. Nevins ${ }^{1}$, Matthew D. Zotta ${ }^{2}$, Richard K. Hailstone ${ }^{1}$, and Eric Lifshin ${ }^{2}$ \\ 1. Rochester Institute of Technology, 54 Lomb Memorial Dr, Rochester, NY, USA \\ 2. SUNY Polytechnic Institute, Albany, NY, USA
}

Astigmatism describes uneven focus in the electron probe of the scanning electron microscope (SEM) which results in distorted, blurred, or stretched images. Factors such as column alignment, the emission pattern from the gun, the lenses, and contamination can cause the desirable circular probe footprint of minimum size to become larger and distorted. Historically, detecting astigmatism relied on going through focus and looking for streaking in any particular direction. The astigmatic beam shape can now be visualized and studied using a modern probe shape determination technique [1-3].

In this study, we investigated a new approach to characterize astigmatism at different beam voltages based on the method found in [1-3]. The following protocol was used at $2 \mathrm{kV}, 5 \mathrm{kV}, 10 \mathrm{kV}$, and $20 \mathrm{kV}$. First, a best focus image was obtained using standard focusing practices, with the aim of achieving the circular beam footprint with minimum diameter. From there, a series of stigmated images were collected by changing the $x$ stigmator alone, the $y$ stigmator alone, or the $x$ and $y$ stigmators. Individually, one stigmator was held constant at the best focus value, while the other was adjusted to the best focus value $\pm 0.2 \%$, $\pm 0.4 \%$, and $\pm 0.6 \%$. Together, the stigmation was adjusted to the four combinations of best focus values $\pm 0.4 \%$ between both stigmators.

To visualize the probe shape, we utilized the point spread function (PSF) determination ability of the Aura Workstation, where the PSF represents the shape of the electron beam. Backscattered electron images were captured with a TESCAN MIRA3 field emission SEM. For the astigmatism series, a calibration sample of $18.2 \mathrm{~nm}$ gold nanoparticles on graphene was imaged for PSF determination purposes. At 10kV, an image of a gold-on-carbon standard was also collected with each beam shape to picture the effect of astigmatism on a sample-of-interest, as well as for testing Aura's image restoration capability.

As the amount of astigmatism increased, the beam became more misshapen (Figure 1). The shape of the astigmatic beam was not necessarily elliptical, and this aspect is now accessible for study. When stigmating the beam along one axis, the shape was not reflected from one side of best focus to the other (especially notable along the $x$ stigmator axis in this case). The behavior shown at $10 \mathrm{kV}$ was mimicked at the other voltages, along with the expected change in diameter of the PSF.

In addition to determining PSFs for the series, image restoration by PSF deconvolution was performed on astigmatic images at $10 \mathrm{kV}$. Overall, the method was able to restore information lost to astigmatism, resulting in improved image clarity. The images captured with highly distorted probe shapes (e.g., $x$ stigmator $=$ best focus $-0.4 \%, y$ stigmator $=$ best focus $+0.4 \%$ ) required more attention and finer tuning of processing parameters when performing restorations to minimize processing artifacts.

Possible applications of astigmatism measurement include characterizing electron focusing elements for a particular microscope, developing or improving an auto-stigmation routine, and as a tool for realizing a basic understanding of how astigmatism affects beam shape in the SEM. 
References:

[1] Lifshin, E., et al., Microscopy Today, 25(3) (2017), p. 18.

[2] Nevins, M., et al., Microscopy \& Microanalysis, 23(S1) (2017), p. 126.

[3] Zotta, M., et al., Microscopy \& Microanalysis, 23(S1) (2017), p. 124.

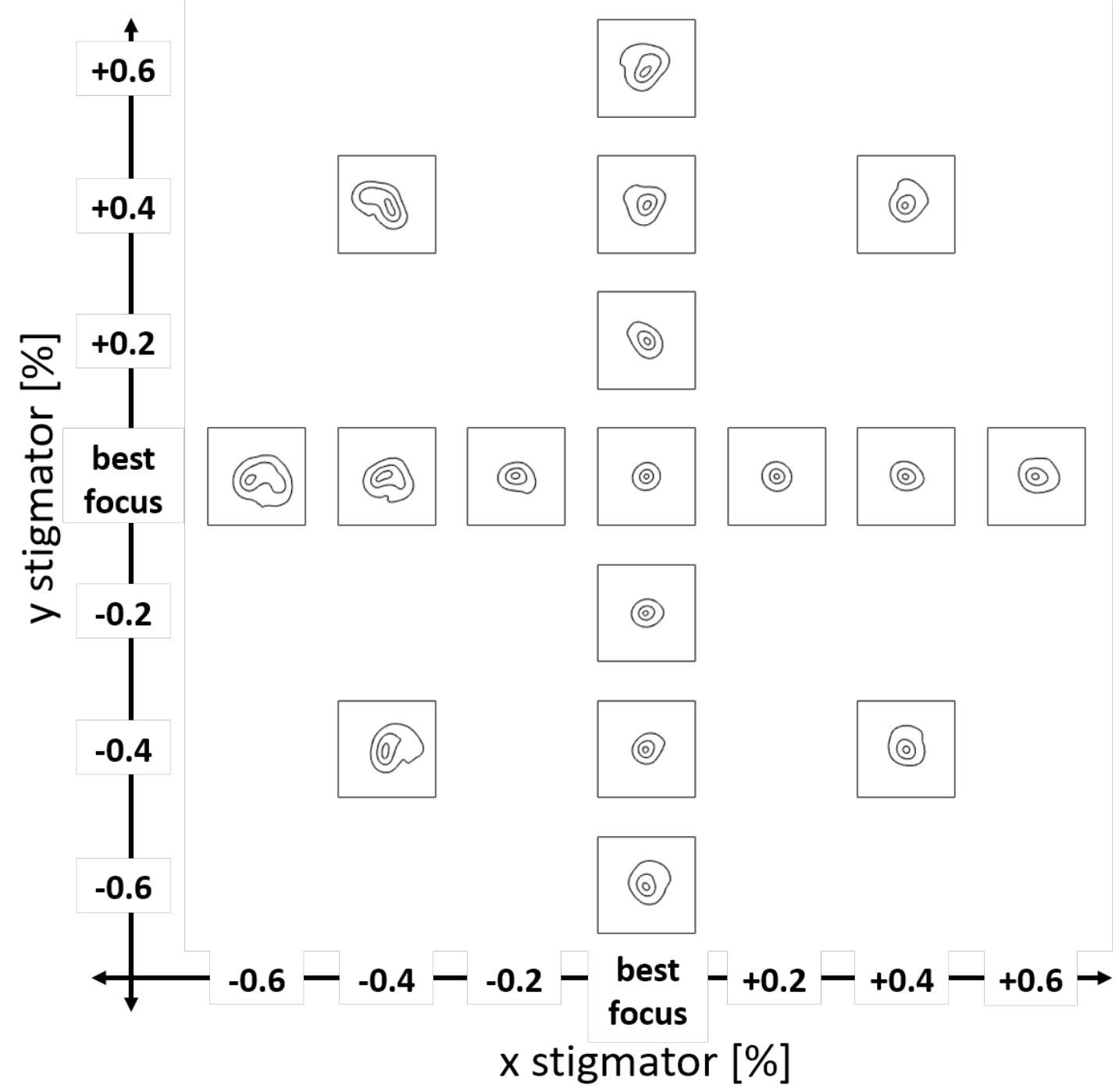

Figure 1. Astigmatism Series at $10 \mathrm{kV}$. Each box contains a contour plot of a point spread function (PSF). The contours shown are $0.9^{*} \max$ (inner), $0.5^{*} \max$ (middle), and $0.1^{*} \max$ (outer), where $\max$ is the maximum intensity of the current PSF. Each PSF was calculated from a calibration image taken with the given stigmator settings. The box size is of scale $85 \mathrm{~nm} \times 85 \mathrm{~nm}$. 